\title{
BMJ Associations of objectively assessed Open levels of physical activity, aerobic fitness and motor coordination with injury risk in school children aged 7-9 years: a cross-sectional study
}

\author{
Eva Martin-Diener, Miriam Wanner, Susi Kriemler, Brian W Martin
}

To cite: Martin-Diener E, Wanner M, Kriemler S, et al. Associations of objectively assessed levels of physical activity, aerobic fitness and motor coordination with injury risk in school children aged 7-9 years: a crosssectional study. BMJ Open 2013;3:e003086.

doi:10.1136/bmjopen-2013003086

- Prepublication history for this paper is available online. To view these files please visit the journal online (http://dx.doi.org/10.1136/ bmjopen-2013-003086).

Received 19 April 2013 Revised 1 July 2013

Accepted 4 July 2013
Physical Activity and Health Unit, Institute of Social and Preventive Medicine, University of Zurich, Zurich, Switzerland

\section{Correspondence to}

Eva Martin-Diener; eva.martin@ifspm.uzh.ch

\section{ABSTRACT}

Objectives: Physical activity (PA) is important for children's health but entails an inherent risk of injuries. The objective of this study was to assess activity-related correlates of injuries in children of the general population under the age of 10 while accounting for PA behaviour objectively assessed with accelerometers.

Design: Cross-sectional.

Setting: Primary schools in Switzerland.

Participants: $41(56.9 \%)$ of 72 contacted schools were eligible. $11(26.9 \%)$ of them agreed to participate. 3 more schools were recruited with a snowball system. On the individual level, $83.7 \%$ of the parents gave consent. Finally, 249 children with complete data $(82.2 \%)$ from 20 grade 1-3 classes from 14 schools were analysed (mean age 7.9 years, $49.4 \%$ girls).

Primary outcome measures: Outcome measures were retrospectively assessed injury incidence rates expressed as the number of injuries per $1000 \mathrm{~h}$ of objectively measured moderate-to-vigorous physical activity (MVPA) and injury risk depending on levels of PA, aerobic fitness and motor coordination, derived from logistic regression models.

Results: 0.43 injuries/1000 h of MVPA ( $95 \% \mathrm{Cl} 0.28$ to 0.58 ) were reported. After controlling for sociodemographic characteristics and accounting for exposure to PA, children with medium and high levels compared with those with low levels of aerobic fitness assessed with the $20 \mathrm{~m}$ shuttle run test were at decreased injury risk (OR=0.37 ( $95 \% \mathrm{Cl} 0.16$ to 0.85$) /$ $\mathrm{OR}=0.29$ (0.16 to 0.63$)$ ). Children with high motor coordination scores assessed with the

"Körperkoordinationstest für Kinder" test were at increased injury risk compared with those in the normal range (2.59 (1.04 to 6.32)). Levels of objectively assessed PA were not associated with injury risk; they were neither expressed as rates nor as cumulative incidence.

Conclusions: This study provides novel data showing that low levels of fitness and high coordinative skills, but not objectively assessed levels of PA, were related to injury risk in children under the age of 10 .

\section{ARTICLE SUMMARY}

Article focus

- Physical activity (PA) is very important for children's health but entails an inherent risk of injuries. However, there are no studies so far investigating the relationship between PA behaviour and the risk of injuries in young children.

- This study focused on describing associations between levels of objectively assessed PA, as well as aerobic fitness and motor coordination, respectively, and injury risk in school children under the age of 10 .

Key messages

- After controlling for exposure to PA and sociodemographic variables, low levels of aerobic fitness and high motor coordination scores were associated with increased injury risk in children aged 7-9 years in the general population.

- Objectively assessed levels of PA were not associated with injury risk in this population.

- There are no indications that fear of injuries should be a reason for avoiding higher levels of PA as such. However, there are first indications that it might be useful to supplement measures aimed at promoting motor skills with those aimed at promoting fitness.

Strengths and limitations of this study

- This study provides novel data: its particular strengths are the assessment of injury risk factors in children of the general population as opposed to child athletes, a study population under the age of 10 and objectively assessed PA exposure data.

- The limitations are the small sample size and the 12-month recall period, which may have resulted in an underestimation of injury incidence rates. In addition, reverse causality cannot be excluded, given the cross-sectional study design.

\section{INTRODUCTION}

The beneficial effects of regular physical activity (PA) on children's physical and 
mental health have been well documented. ${ }^{1}$ However, being physically active entails an inherent risk of injuries. Injuries in children are generally less severe than in adults. ${ }^{2}$ However, they are frequent with cumulative incidences over 1 year for sports and activity-related injuries treated by medical professionals varying between about $5 \%$ and $15 \% .^{2}{ }^{3}$ Therefore, injuries represent a relevant public health and economic burden. ${ }^{4}$ Owing to the current low activity levels in children and adolescents, ${ }^{5}$ there are many efforts to increase PA, fitness or motor skills in youth. So far, little attention has been paid to the question whether more injuries have to be expected with increasing levels of PA or whether higher levels of activity, better fitness or motor skills could prevent children from getting injured either while being physically active or even in other contexts.

Not only are intervention studies assessing the effects of PA promotion on injury risk in children lacking, but also observational data on injury risk and activity-related correlates of injuries in young children of the general population are scarce. ${ }^{6}$ An Australian study investigated sports and activity-related injury incidence rates (IIRs) in 5 -year-olds to 12 -year-olds, ${ }^{7}$ and a Dutch study did so in school children aged 10-12 years. ${ }^{8}$ The latter seems to be the first study that also assessed IIRs for children according to different levels of PA. ${ }^{9}$ Studies on associations between different measures of motor coordination and cumulative injury incidence (CII) are scarce and inconclusive. Some found that more skilled children had more injuries, ${ }^{10}{ }^{11}$ whereas others reported more injuries for less skilled individuals ${ }^{12}$ or found no associations. ${ }^{13}$ While these studies had not taken exposure to PA into account, the aforementioned Dutch study did so, and it found no associations between injury rates and levels of motor coordination. ${ }^{9}$ Regarding aerobic fitness in youth, suggestions for inverse associations with sports injuries ${ }^{14}$ but direct associations with fractures ${ }^{15}$ were found.

To our knowledge, there is no study so far which has investigated IIRs in children of the general population under the age of 10 years, taking into account levels of PA, motor coordination and aerobic fitness simultaneously. The purpose of this cross-sectional analysis was to describe the role of objectively assessed levels of PA, sports behaviour, motor coordination and aerobic fitness as correlates of injuries related to PA or other causes, in school children aged 7-9 years.

\section{METHODS}

\section{Recruitment and population}

For this study, data of the baseline assessment of a quasi-experimental intervention study on the effects of a national school-based PA intervention programme were used; the necessary sample size had been estimated in order to investigate those intervention effects. Schools were selected in a random-based multistep procedure in cooperation with the public administration of the Swiss
Canton of Aargau in autumn/winter 2011/2012. The inclusion criteria were that participating teachers had not yet undergone specific training to teach extracurricular PA and sports in the context of the national programme under investigation, that they taught first, second or third graders and that they were going to keep the same class in the school year 2012/2013. In total, 72 schools were contacted; $31(43.1 \%)$ did not meet the inclusion criteria. Nineteen $(46.3 \%)$ of the eligible schools declined to participate, five $(12.2 \%)$ could not be reached and six $(14.6 \%)$ could be reached but did not answer back. Overall, $11(26.9 \%)$ of the eligible schools participated in the study, two among them with two classes each, yielding a total of 13 classes. In addition, one class joined the baseline assessments only. Finally, 6 more classes from 3 additional schools were recruited with a snowball system, totalling 20 classes from 14 schools.

The study was approved by the Ethics Committee of the Canton of Aargau, Switzerland. In each class, parents were invited to an information event where the goals and methods of the study were presented. Active parental consent was obtained using an information sheet for the parents, one for the child and a consent form.

\section{Assessments and definitions Procedure}

In each class, motor coordination and aerobic fitness were assessed during a lesson of physical education. After these assessments, accelerometers were distributed to the children. Each child was instructed by a member of the research team on how to correctly use the device. Furthermore, the children received a parental questionnaire to take home and then back to school in a sealed envelope. After 1 week, the accelerometers and questionnaires were gathered by the teacher and collected in the schools by a member of the research team.

\section{Assessment and definition of correlates of injuries}

PA behaviour was assessed objectively using accelerometers (Actigraph GT3X and GT3X+, Pensacola, Florida, USA) and a $5 \mathrm{~s}$ epoch time was chosen. The children wore the devices on the right hip for seven consecutive days during waking hours; during waterbased activities, the devices had to be removed. The software ActiLife 6 was used to screen, clean and analyse the data. At least four valid measurement days (including at least one weekend day/at least $8 \mathrm{~h}$ of recorded activity time per day) were required for inclusion of the child. For these analyses, minutes of moderate-to-vigorous intensity physical activity (MVPA) per day were calculated using published cut points, ${ }^{16}$ and children were grouped into age-specific and sexspecific tertiles.

The weekly frequency of trainings in a sports club was assessed with the parental questionnaire. For the 
purpose of this analysis, children were categorised into two groups (sports club attendance yes/no).

The classes performed the body coordination test by Kiphard-Schilling (Körperkoordinationstest für Kinder $(\mathrm{KTK}))^{17}$ and then the $20 \mathrm{~m}$ shuttle run test. ${ }^{18}$ Each test element of KTK (balancing backward, jumping laterally, shifting platforms, hopping on one leg over an obstacle) was supervised by at least one researcher. The children changed from test element to element in groups of three to five. The age-specific and sex-specific standardised KTK motor quotient (MQ) scores were calculated and children were categorised according to the manual into the standard groups 'normal' (86-115 points), 'low' (less than 86 points) and 'high' (more than 115 points). After the KTK test, the children performed the shuttle run. For these analyses, the number of $20 \mathrm{~m}$ laps performed was used and children were grouped into agespecific and sex-specific tertiles. During the KTK and shuttle run testing procedure, height and body weight were measured and body mass index was calculated $\left(\mathrm{BMI}\right.$, in $\mathrm{kg} / \mathrm{m}^{2}$ ). To define overweight, age-specific and sex-specific cut points were used. ${ }^{19}$

Parental education and place of birth were assessed in the parental questionnaire. High parental education was defined as having a father or a mother with a tertiary degree. Children were classified as having a migrational background if at least one parent had been born abroad. $^{20}$

\section{Definition and assessment of injuries}

An injury was to be reported if it had been treated by a medical professional or a guardian and if the child could not continue with his or her activity for the rest of the day. The number of injuries as well as their nature, location and context were assessed retrospectively for the past 12 months in the parental questionnaire. If the child had been injured more than once, parents were asked to report those details for the most severe injury.

\section{Statistical analyses}

Statistical analyses were performed using STATAV.12.1 statistical software. CII is reported as the percentage of the respective population injured in the past 12 months. IIRs with $95 \%$ CIs are expressed as the number of injuries per $1000 \mathrm{~h}$ of exposure to MVPA. To estimate exposure to MVPA during 12 months, the mean daily minutes of MVPA assessed by accelerometers during the measurement week were extrapolated to 1 year. To assess the role of different potential correlates of injury risk, ORs with 95\% CI were estimated using univariate and multivariate logistic regression models adjusted for clustering within the classes. In an additional step, a potential interaction between levels of aerobic fitness and levels of motor skills was tested. Finally, subgroup analyses were conducted for children with low levels and children with medium/high levels of aerobic fitness, respectively.

\section{RESULTS}

\section{Study population}

Of a total of 362 children and parents, 303 parents $(83.7 \%)$ consented to participate in the study. Valid accelerometer data were available from 287 children (94.7\% of those consenting). Another 38 observations were dropped due to missing values for fitness, KTK or questionnaire data. The final sample thus comprised 249 children $(82.2 \%$ of those consenting). One hundred and twenty-six were boys $(50.6 \%)$ and the mean age was 7.9 years. Further characteristics of the study population are given in table 1 .

\section{Description of injuries}

For 38 children $(15.3 \%)$, a total of 54 injuries were reported for the 12 months preceding the assessment. Twenty-five children had been injured once, 10 two times and 3 three times. For those children with more than one injury, detailed information was provided only for the most severe event. More than $90 \%$ of these injuries were associated with some kind of PA: more than half of the children were injured while playing, walking or running around and another third named a specific activity (table 2).

\section{Correlates of injuries}

CII over the past 12 months, as well as IIR by potential correlates of the study population, is presented in table 3 . CII tended to be higher among older children

\section{Table 1 Description of study population ( $\mathrm{N}=249)$}

Total N (\%) Boys N (\%) Girls N (\%)

Age

\begin{tabular}{|c|c|c|c|}
\hline$<8$ years & $176(70.7)$ & $84(66.7)$ & $92(74.8)$ \\
\hline$\geq 8$ years & $73(29.3)$ & $42(33.8)$ & $31(25.2)$ \\
\hline \multicolumn{4}{|c|}{ arental education } \\
\hline Low & $134(53.8)$ & 73 (57.9) & $61(49$ \\
\hline High & 115 (46.2) & $53(42.1)$ & $62(50$ \\
\hline \multicolumn{4}{|c|}{ Aigrational background } \\
\hline No & $143(57.4)$ & $76(60.3)$ & $67(54$ \\
\hline Yes & $106(42.6)$ & $50(39.7)$ & $56(4$ \\
\hline \multicolumn{4}{|l|}{ verweight } \\
\hline No & $204(81.9)$ & $102(80.9)$ & $102(82$ \\
\hline Yes & $45(18.1)$ & $24(19.1)$ & $21(17$ \\
\hline \multicolumn{4}{|c|}{ KTK motor quotient } \\
\hline Low & $19(7.6)$ & $7(5.6)$ & $12(9.8$ \\
\hline Normal & $156(62.7)$ & $70(55.6)$ & $86(6 \varsigma$ \\
\hline High & 74 (29.7) & 49 (38.9) & $25(2 C$ \\
\hline \multicolumn{4}{|c|}{ raining in a sports club } \\
\hline No & $112(45.0)$ & $40(31.8)$ & $72(5$ \\
\hline \multirow[t]{2}{*}{ Yes } & $137(55.0)$ & $86(68.2)$ & $51(41$ \\
\hline & Mean (SD) & Mean (SD) & Mean (SD \\
\hline
\end{tabular}

$\begin{array}{llll}\text { MVPA (min) } & 82.5(22.3) & 93.2(21.9) & 71.4(16.6)\end{array}$ Shuttle run test $\quad 35.8(16.0) \quad 40.7(16.8) \quad 30.8(13.4)$ (number of $20 \mathrm{~m}$ laps) 


\begin{tabular}{lc} 
Table 2 Description of injuries $(\mathrm{N}=38)$ & \\
\hline & $\mathrm{N}(\%)$ \\
\hline Activity when injured & \\
Distinct activity (ie, cycling, skiing, playing & $13(34)$ \\
football or another sport) & \\
Playing (indoors, outdoors) & $16(42)$ \\
Walking, running (not in sports) & $6(16)$ \\
Other & $2(5)$ \\
Missing & $1(3)$ \\
Type of injury & $7(18)$ \\
Bruise, abrasion & $13(34)$ \\
Laceration, cut & $5(13)$ \\
Ligament sprain, luxation, muscle strain & $6(16)$ \\
Fracture & $2(5)$ \\
Concussion, back and neck problems & $3(8)$ \\
Other & $2(5)$ \\
Missing & \\
Injury location & $14(37)$ \\
Head & $1(3)$ \\
Neck, trunk & $9(24)$ \\
Upper extremities & $11(29)$ \\
Lower extremities & $3(8)$ \\
More than one/missing & $12(32)$ \\
Treatment of injury & $26(68)$ \\
Parent or other guardian & $11(29)$ \\
Medical professional & $13(34)$ \\
Not able to be active as usual after being injured & $13(34)$ \\
Current day & \\
1-7 days & \\
8 days or longer & \\
Missing & \\
\hline & \\
\hline
\end{tabular}

$(\mathrm{p}=0.060)$. After taking PA exposure into account, girls had higher IIR than boys $(\mathrm{p}=0.032)$. IIR was higher in children with the lowest levels of aerobic fitness compared with those with medium levels $(\mathrm{p}=0.027)$, and there was a trend towards significance when the lowest level was compared with the highest level of aerobic fitness ( $p=0.076$ ). Furthermore, IIR tended to be higher in children with high motor skills compared with those in the normal range $(p=0.103)$. However, higher levels of objectively assessed PA were not associated with increased injury incidence, neither for CII nor for IIR. Children attending a sports club had higher CII and IIR than their counterparts, but not at statistically significant levels.

Logistic regression models adjusted for clustering within classes were used to assess the risk of having had an injury depending on potential correlates. In the univariate model and in the multivariate model-with all associations controlled for exposure to PA and for other variables in the model-older children tended to be at higher risk for injuries. No other sociodemographic variable seemed to be associated with injury risk (table 4). However, children in the second and third tertiles of aerobic fitness were at lower injury risk compared with children in the lowest tertile, and children with a high compared with a normal MQ were at increased injury risk, even after controlling for PA exposure. Again, after controlling for other variables, more active children were not at increased injury risk compared with inactive children. For those attending a sports club, ORs indicated an elevated injury risk, even after adjusting for PA, though not at statistically significant levels. The model was also run with age and BMI as continuous variables and the results were comparable.

The multivariate logistic model described in table 4 was also run only for injuries treated by a health professional. For the correlates described above, there were no substantial differences, though as expected estimates were less precise and only reached the level of statistical significance for the third tertile in the shuttle run test.

\section{Subgroup analysis and interactions}

To investigate interactions between aerobic fitness and motor coordination, the second and the third tertiles of aerobic fitness were collapsed into one category. CII in children with high motor coordination but low aerobic fitness was $44.4 \%$ while CII in children with high motor coordination and medium/high aerobic fitness was only $15.4 \%$. In a subgroup analysis, the multivariate logistic model presented in table 4 was run separately for children with low aerobic fitness and for children with medium/high aerobic fitness. In children with low fitness, those with high motor skills had a dramatically increased injury risk compared with those in the normal range $(\mathrm{OR}=8.04$ (3.11 to $20.80, \mathrm{p}<0.001)$. In children with medium/high aerobic fitness, injury risk tended to be elevated in those with high motor skills but not at statistically significant levels $(\mathrm{OR}=2.03$ (0.81 to 5.13), $\mathrm{p}=0.133)$. To test an interaction term in the logistic model, the two motor coordination categories 'low' and 'normal' were also collapsed. In the modified model including an interaction term of the two now binary variables for motor coordination and aerobic fitness, the main effects remained statistically significant (high vs low/normal motor coordination: $\mathrm{OR}=4.66 \quad(1.6$ to 13.01), $\mathrm{p}=0.003$; medium/high vs low aerobic fitness: $\mathrm{OR}=0.36$ (0.13 to 0.99$), \mathrm{p}=0.049)$. The interaction effect was considerable but did not reach statistical significance $(\mathrm{OR}=0.46(0.12$ to 1.71$), \mathrm{p}=0.248)$.

\section{DISCUSSION}

This cross-sectional study was conducted in school children aged 7-9 years and it found that low levels of aerobic fitness and high levels of motor skills were associated with increased injury risk after accounting for exposure to objectively assessed PA. Levels of PA were not associated with injury risk.

The strengths of our study are that injury risk factors in children focusing on the general population, but not on an athlete population, were assessed and that exposure data were based on objectively assessed PA. Furthermore, the study contributes to answering the open question of the relationship between motor coordination and injuries in primary school children while 
Table 3 Cumulative injury incidence (CII; in \%) and injury incidence rates (IIRs; with 95\% Cl) in different subgroups (N=249)

\begin{tabular}{|c|c|c|c|c|}
\hline & CII (\%) & p Value* & IIR (95\% CI)† & p Value* \\
\hline Overall & 15.3 & & $0.43(0.28$ to 0.58$)$ & \\
\hline \multicolumn{5}{|l|}{ Sex } \\
\hline Boys & 15.1 & \multirow[t]{2}{*}{0.936} & $0.32(0.17$ to 0.47$)$ & \multirow[t]{2}{*}{0.032} \\
\hline Girls & 15.5 & & $0.58(0.31$ to 0.86$)$ & \\
\hline \multicolumn{5}{|l|}{ Age } \\
\hline$<8$ years & 12.5 & \multirow[t]{2}{*}{0.060} & $0.38(0.20$ to 0.55$)$ & \multirow[t]{2}{*}{0.177} \\
\hline$\geq 8$ years & 21.9 & & $0.56(0.27$ to 0.84$)$ & \\
\hline \multicolumn{5}{|c|}{ Parental education } \\
\hline Low & 14.9 & \multirow[t]{2}{*}{0.874} & $0.41(0.22$ to 0.60$)$ & \multirow[t]{2}{*}{0.648} \\
\hline High & 15.7 & & $0.46(0.22$ to 0.70$)$ & \\
\hline \multicolumn{5}{|c|}{ Migrational background } \\
\hline No & 16.8 & \multirow[t]{2}{*}{0.438} & $0.47(0.28$ to 0.67$)$ & \multirow[t]{2}{*}{0.395} \\
\hline Yes & 13.2 & & $0.37(0.14$ to 0.60$)$ & \\
\hline \multicolumn{5}{|l|}{ Overweight } \\
\hline No & 14.2 & \multirow[t]{2}{*}{0.329} & $0.40(0.25$ to 0.55$)$ & \multirow[t]{2}{*}{0.253} \\
\hline Yes & 20.0 & & $0.59(0.11$ to 1.06$)$ & \\
\hline \multicolumn{5}{|c|}{ Minutes of MVPA } \\
\hline $\mathrm{T} 1$ & 14.0 & \multirow[t]{3}{*}{0.916} & $0.48(0.17$ to 0.79$)$ & T1 vs T2: 0.801 \\
\hline $\mathrm{T} 2$ & 15.9 & & $0.44(0.18$ to 0.70$)$ & T2 vs T3: 0.695 \\
\hline T3 & 16.1 & & $0.39(0.17$ to 0.61$)$ & T3 vs T1: 0.525 \\
\hline \multicolumn{5}{|c|}{ KTK motor quotient } \\
\hline Low & 21.1 & \multirow[t]{3}{*}{0.372} & $0.49(0.00$ to 0.98$)$ & Low vs normal: 0.527 \\
\hline Normal & 12.8 & & $0.35(0.17$ to 0.53$)$ & Normal vs high: 0.103 \\
\hline High & 18.9 & & 0.57 (0.26 to 0.87$)$ & High vs low: 0.837 \\
\hline \multicolumn{5}{|c|}{ Shuttle run test (number of $20 \mathrm{~m}$ laps) $\ddagger$} \\
\hline $\mathrm{T} 1$ & 22.1 & \multirow[t]{3}{*}{0.086} & $0.64(0.32$ to 0.96$)$ & T1 vs T2: 0.027 \\
\hline T2 & 10.6 & & 0.30 (0.09 to 0.52$)$ & T2 vs T3: 0.331 \\
\hline T3 & 12.8 & & $0.36(0.13$ to 0.59$)$ & T3 vs T1: 0.076 \\
\hline \multicolumn{5}{|c|}{ Training in sports club } \\
\hline No & 11.6 & \multirow[t]{2}{*}{0.147} & $0.35(0.12$ to 0.58$)$ & \multirow[t]{2}{*}{0.239} \\
\hline Yes & 18.3 & & 0.49 (0.29 to 0.69$)$ & \\
\hline
\end{tabular}

taking into account exposure to PA. Participation was high and, compared with other studies, ${ }^{21}$ compliance with wearing accelerometers was excellent. Our study represents the range of exposures typically found in a population of Swiss primary school children and can be considered as generalisable to a multicultural Western European population. Our sample was selected based on criteria independent of injury risk or potential injury correlates. This study also has some limitations. The sample size was small. Only larger sample sizes would allow ruling out smaller effects such as the role of sports club participation for injury risk. In order to collect a larger number of injuries even in our relatively small sample (and in analogy to the HBSC survey, ${ }^{22}$ ) we chose a 12-month recall period. We were aware that such a long recall frame may result in an underestimation of injury incidences. ${ }^{23}$ However, in our study, there was no indication that under-reporting was different with respect to exposure. To increase numbers and thus power, we also collected some injuries that were treated by laypersons and not medical professionals. An analysis including only injuries treated by health professionals showed essentially unchanged but less precise effect estimates. Therefore, we are confident that the associations observed in our study are valid, though the frequency of injuries might differ from those in other studies. With the retrospective assessment of injuries, reverse causality may have occurred, that is, children could have been less active because of an existing injury. However, it is rather unlikely that, owing to a recent injury, a significant number of children were less active than usual in the particular measurement week. Even though participation was good, some of the participants were lost during the study. We expect that this loss was mainly non-differential with respect to the associations between the correlates of interests and injuries and therefore did not result in substantial bias. Finally, PA was assessed during 1 week and extrapolated for the past 12 months to estimate exposure.

Owing to the different methodologies and definitions of injuries, comparability with other studies is limited. The vast majority of injuries in our population occurred 
Table 4 Logistic regression analyses for the risk of having been injured in the past 12 months ( $N=249)$

\begin{tabular}{|c|c|c|}
\hline & $\begin{array}{l}\text { Univariate } \\
\text { analysis* } \\
\text { OR }(95 \% \mathrm{Cl})\end{array}$ & $\begin{array}{l}\text { Multivariate } \\
\text { analysis }{ }^{\star} \dagger \\
\text { OR }(95 \% \mathrm{Cl})\end{array}$ \\
\hline \multicolumn{3}{|l|}{ Sex } \\
\hline Boys & 1 & 1 \\
\hline Girls & 1.03 (0.53 to 2.00$)$ & 1.58 (0.65 to 3.83$)$ \\
\hline \multicolumn{3}{|l|}{ Age } \\
\hline$<8$ years & 1 & 1 \\
\hline$\geq 8$ years & $1.96(0.94$ to 4.11$)$ & 1.84 (0.98 to 3.49$)$ \\
\hline \multicolumn{3}{|c|}{ Parental education } \\
\hline Low & 1 & 1 \\
\hline High & $1.06(0.65$ to 1.72$)$ & $1.23(0.70$ to 2.15$)$ \\
\hline \multicolumn{3}{|c|}{ Migrational background } \\
\hline No & 1 & 1 \\
\hline Yes & $0.75(0.34$ to 1.70$)$ & 0.75 (0.32 to 1.77$)$ \\
\hline \multicolumn{3}{|c|}{ Overweight } \\
\hline No & 1 & 1 \\
\hline Yes & $1.51(0.51$ to 4.48$)$ & $1.48(0.43$ to 5.11$)$ \\
\hline \multicolumn{3}{|c|}{ Minutes of MVPA } \\
\hline $\mathrm{T} 1$ & 1 & 1 \\
\hline T2 & $1.16(0.64$ to 2.11$)$ & 1.05 (0.52 to 2.14$)$ \\
\hline T3 & $1.18(0.62$ to 2.23$)$ & $1.22(0.58$ to 2.56$)$ \\
\hline \multicolumn{3}{|c|}{ KTK motor quotient } \\
\hline Low & $1.81(0.44$ to 7.50$)$ & $1.29(0.30$ to 5.51$)$ \\
\hline Normal & 1 & 1 \\
\hline High & $1.59(0.78$ to 3.21$)$ & 2.59 (1.04 to 6.32$)$ \\
\hline \multicolumn{3}{|c|}{ Shuttle run test (number of $20 \mathrm{~m}$ laps) $\ddagger$} \\
\hline $\mathrm{T} 1$ & 1 & 1 \\
\hline T2 & $0.43(0.18$ to 1.03$)$ & $0.37(0.16$ to 0.85$)$ \\
\hline T3 & $0.48(0.29$ to 0.77$)$ & $0.29(0.16$ to 0.63$)$ \\
\hline \multicolumn{3}{|c|}{ Training in a sports club } \\
\hline No & 1 & 1 \\
\hline Yes & $1.70(0.81$ to 3.55$)$ & $1.77(0.76$ to 4.10$)$ \\
\hline \multicolumn{3}{|c|}{$\begin{array}{l}\text { *Adjusted for clustering within classes. } \\
\text { †lncludes all variables in the model. } \\
\text { †T1-T3: sex-specific and age-specific tertiles generated from the } \\
\text { study population (T1=lowest tertile). KTK, Körperkoordinationstest } \\
\text { für Kinder; MVPA, moderate-to-vigorous intensity physical activity. }\end{array}$} \\
\hline
\end{tabular}

while the children were physically active, mostly during active play or while walking or running around. This finding is consistent with another study. ${ }^{7}$ In our study and in a Dutch study, ${ }^{8}$ CII was comparable, ranging from $13 \%$ to $15 \%$ over a 1 year period. Our IIR of 0.43 injuries $/ 1000 \mathrm{~h}$ of activity was also comparable to the Dutch and Australian rates of $0.48-0.50 .{ }^{78}$ Higher age tended to be associated with increased injury risk, which is consistent with other studies in the general population ${ }^{9}$ or in youth athletes. ${ }^{14}$ Regarding IIR, girls were at a higher risk than boys, which is also consistent with other findings. ${ }^{9}$ Studies investigating associations between aerobic fitness, motor skills and injury risk in young children of the general population are scarce. Our finding that high fitness might protect against injuries is consistent with results from studies in youth athlete populations. ${ }^{14}$ However, in a recent study in youth, the lifetime cumulative incidence of fractures was directly associated with aerobic fitness, but only in those children with low grip strength. ${ }^{15}$ The general public perception is that less skilled children are at a higher injury risk. Yet, we found the opposite scenario, that this was true for the most skilled children, even after accounting for exposure to PA. There are indications that this was particularly the case for children with good motor coordination but low aerobic fitness. We can only speculate that these children might not be physically prepared for some coordination tasks that they are able to perform, or that skilled children in general might be inclined to take the highest risks and literally 'climb the highest trees'. There are a few studies investigating associations between motor coordination and injuries in primary school children. In a publication from 2003, ${ }^{13}$ the authors have described the findings as mixed. Their conclusion was based on early reports which had shown direct ${ }^{11}$ or inverse ${ }^{12}$ associations between motor coordination and injuries. In their own study, the authors found no association between motor ability scores and retrospectively assessed lifetime CII of injuries. ${ }^{13}$ In a later study, others found direct associations for some, but not all, measures of motor coordination with cumulative incidence of prospectively assessed school injuries. ${ }^{10}$ The only study so far reporting injury risk adjusted for exposure to PA found no association between motor coordination scores and prospectively assessed PA-related injuries. ${ }^{9}$ After the inclusion of our study results, the limited evidence available so far suggests no association or a direct association rather than an inverse relationship between motor coordination and injuries in school children.

We were not able to perform analyses of prospective injury incidence for which another study reported that the most inactive children were at the highest injury risk. ${ }^{9}$ In studies in adolescents, sports club attendance was associated with an increased CII for sports injuries, ${ }^{22}$ and also overall injuries after controlling for other risk behaviours. ${ }^{24}$ In our study, associations between sports club participation and injury risk also showed considerable effect size estimates, but not at statistically significant levels. Whether this less clear relationship in our sample is due to the younger age group with possibly less risk-seeking behaviour among sport-active individuals than in an adolescent population or whether this observation has simply to be attributed to the smaller power of our study is not clear.

In our study, retrospectively assessed injury risk was associated with low fitness and high motor coordination scores, but not with levels of PA. Studies with higher power and prospective assessments of injuries will be necessary to further explore activity-related risk for injuries and protective factors in young school children. In future steps, effects of PA interventions on injury risk will need to be investigated. If confirmed, our findings have implications for PA promotion programmes in primary school children, suggesting the inclusion of measures aimed not only at promoting motor skills, but also fitness and possibly other measures of injury prevention. PA interventions that have 
the potential to also decrease and not increase injury risk in children should be developed and tested.

Acknowledgements We thank Christian Koch and Christian Müller, Canton of Aargau, for their support regarding recruitment; Nadine Eberle, Sabrina Eibisch, Claudia Frick, Michèle Geissbühler, Theo Härry, Pascal Hostettler, Melanie Keller, Mirco Trachsel and Claudia Wintersohle for their assistance in data collection. And we also thank all teachers, headmasters, children and parents for their participation in the study.

Contributors All four authors have contributed to the design of this study. BWM was the principal investigator. EM-D conducted the analyses, supported by MW and BWM, and also drafted the paper. MW and EM-D were responsible for data collection, supported by SK. All authors contributed to the critical revision of the manuscript and read and approved the final version.

Funding Swiss Research Concept Sports and Physical Activity 2008-2011 (grant 11-05), Swiss Council for Accident Prevention, Migros culture percentage, Swiss Foundation for the Health of Children and Adolescents and Swiss Association of Sports Medicine.

Competing interests None.

Ethics approval Ethics Committee of the Canton of Aargau, Switzerland.

Provenance and peer review Not commissioned; externally peer reviewed.

Data sharing statement No additional data are available.

Open Access This is an Open Access article distributed in accordance with the Creative Commons Attribution Non Commercial (CC BY-NC 3.0) license, which permits others to distribute, remix, adapt, build upon this work noncommercially, and license their derivative works on different terms, provided the original work is properly cited and the use is non-commercial. See: http:// creativecommons.org/licenses/by-nc/3.0/

\section{REFERENCES}

1. U.S. Department of Health and Human Services. Physical Activity Guidelines Advisory Committee Report. In: Services USDoHaH, ed. Washington, 2008.

2. Swiss council for Accident Prevention. Statistics on non-occupational accidents and the level of safety in Switzerland, Road traffic, sports, home and leisure. Bern: BFU, 2012.

3. Collard DC, Verhagen EA, Chin APMJ, et al. Acute physical activity and sports injuries in children. Appl Physiol Nutr Metab 2008:33:393-401.

4. Collard DC, Verhagen EA, van Mechelen W, et al. Economic burden of physical activity-related injuries in Dutch children aged 10-12. Br $J$ Sports Med 2011;45:1058-63.

5. Hallal PC, Andersen LB, Bull FC, et al. Global physical activity levels: surveillance progress, pitfalls, and prospects. Lancet 2012;380:247-57.
6. Mytton J, Towner E, Brussoni M, et al. Unintentional injuries in school-aged children and adolescents: lessons from a systematic review of cohort studies. Inj Prev 2009;15:111-24.

7. Spinks $A B$, Macpherson $A K$, Bain $C$, et al. Injury risk from popular childhood physical activities: results from an Australian primary school cohort. Inj Prev 2006;12:390-4.

8. Verhagen E, Collard D, Paw MC, et al. A prospective cohort study on physical activity and sports-related injuries in 10-12-year-old children. Br J Sports Med 2009;43:1031-5.

9. Bloemers F, Collard D, Paw MC, et al. Physical inactivity is a risk factor for physical activity-related injuries in children. $\mathrm{Br} J$ Sports Med 2012;46:669-74.

10. Gofin R, Donchin M, Schulrof B. Motor ability: protective or risk for school injuries? Accid Anal Prev 2004;36:43-8.

11. Langley J, Silva PA, Williams SM. Motor coordination and childhood accidents. J Safety Res 1980;12:175-8.

12. Angle CR. Locomotor skills and school accidents. Pediatrics 1975;56:819-22.

13. Schwebel DC, Binder SC, Sales JM, et al. Is there a link between children's motor abilities and unintentional injuries? J Safety Res 2003;34:135-41.

14. Emery CA. Risk factors for injury in child and adolescent sport: a systematic review of the literature. Clin J Sport Med 2003;13:256-68.

15. Clark EM, Tobias JH, Murray L et al. Children with low muscle strength are at an increased risk of fracture with exposure to exercise. J Musculoskelet Neuronal Interact 2011;11:196-202.

16. Evenson KR, Catellier DJ, Gill K, et al. Calibration of two objective measures of physical activity for children. $J$ Sports Sci 2008;26:1557-65.

17. Kiphard EJ, Schilling F. Körperkoordinationstest für Kinder KTK. Manual. 2. überarbeitete und ergänzte Auflage. Göttingen: Belz, 2007.

18. Council of Europe. Eurofit. Handbook for the Eurofit tests of physical fitness. Rome: Committee for the development of sport, 1988.

19. de Onis M, Onyango AW, Borghi E, et al. Development of a WHO growth reference for school-aged children and adolescents. Bull World Health Organ 2007;85:660-7.

20. Bundesamt fürStatistik. Ein Drittel der Schweizer Bevölkerung sind Migranten oder Nachkommen von Migranten. Pressemitteilung $\mathrm{Nr}$. 0350-0209-60. Neuenburg, 2002

21. Verloigne M, Van Lippevelde W, Maes L, et al. Levels of physical activity and sedentary time among 10- to 12-year-old boys and girls across 5 European countries using accelerometers: an observationa study within the ENERGY-project. Int J Behav Nutr Phys Act 2012;9:34.

22. Michaud PA, Renaud A, Narring F. Sports activities related to injuries? A survey among 9-19-year-olds in Switzerland. Inj Prev 2001;7:41-5.

23. Harel $\mathrm{Y}$, Overpeck MD, Jones $\mathrm{DH}$, et al. The effects of recall on estimating annual nonfatal injury rates for children and adolescents. Am J Public Health 1994;84:599-605.

24. Mattila V, Parkkari J, Kannus P, et al. Occurrence and risk factors of unintentional injuries among 12- to 18-year-old Finns-a survey of 8219 adolescents. Eur J Epidemiol 2004;19:437-44. 